\title{
Transmissibility of coronavirus disease 2019 in Chinese cities with different dynamics of imported cases
}

\author{
Ka Chun Chong ${ }^{1,2}$, Wei Cheng ${ }^{3}$, Shi Zhao ${ }^{1,2}$, Feng Ling ${ }^{3}$, Kirran N Mohammad ${ }^{1}$, Maggie Wang ${ }^{1,2}$, Benny CY Zee $^{1,}$ \\ ${ }^{2}$, Lai Wei ${ }^{1}$, Xi Xiong ${ }^{1}$, Hengyan Liu ${ }^{1}$, Jingxuan Wang ${ }^{\text {Corresp., } 1}$, Enfu Chen ${ }^{\text {Corresp. } 3}$ \\ ${ }^{1}$ School of Public Health and Primary Care, The Chinese University of Hong Kong, Hong Kong SAR, China \\ 2 Shenzhen Research Institute, The Chinese University of Hong Kong, Shenzhen, China \\ 3 Zhejiang Province Centre for Disease Control and Prevention, Hangzhou, China \\ Corresponding Authors: Jingxuan Wang, Enfu Chen \\ Email address: jxwang@link.cuhk.edu.hk, enfchen@cdc.zj.cn
}

Background: Monitoring the reproduction number $\left(R_{t}\right)$ of the disease could help determine whether there is sustained transmission in a population, but areas with similar epidemic trends could have different transmission dynamics given the risk from imported cases varied across regions. In this study, we examined the $R_{t}$ of coronavirus disease 2019 (COVID-19) by taking different dynamics of imported cases into account and compared the transmissibility of COVID-19 at different intervention periods in Hangzhou and Shenzhen.

Methods: We obtained the daily aggregated counts of laboratory-confirmed imported and local cases of COVID-19 infections in Hangzhou and Shenzhen from January 1 to March 13, 2020. Daily $R_{t}$ and piecewise $R_{t}$ before and after Wuhan lockdown were estimated, accounting for imported cases.

Results: Since the epidemic of COVID-19 in Shenzhen was dominated by imported cases, $R_{t}$ was around 0.1 to 0.7 before the Wuhan lockdown. After the lockdown of Wuhan and the initialization of measures in response to the outbreak, local transmission was well-controlled as indicated by a low estimated value of piecewise $R_{t}, 0.15$ (95\% Cl: 0.09 to 0.21 ). On the contrary, $R_{t}$ obtained for Hangzhou ranged from 1.2 to 4.9 with a piecewise $R_{t}$ of 2.55 (95\% Cl: 2.13 to 2.97$)$ before the lockdown of Wuhan due to the surge in local cases. Credits to the Wuhan lockdown and other outbreak response measures, $R_{t}$ dropped below unity in mid-February.

Conclusions: Even though Shenzhen had more cases than Hangzhou, local transmission did not sustain probably due to limited transmission from imported cases owing to the reduction in local susceptibles as residents left the city during Chunyun. The lockdown measures and local outbreak responses helped reduce the local transmissibility. 
1 Transmissibility of coronavirus disease 2019 (COVID-19) in Chinese cities with different

2 dynamics of imported cases

3 Ka Chun Chong ${ }^{1,2}$, Wei Cheng ${ }^{3}$, Shi Zhao ${ }^{1,2}$, Feng Ling ${ }^{3}$, Kirran N. Mohammad ${ }^{1}$, Maggie Haitian

4 Wang $^{1,2}$, Benny Chung Ying Zee ${ }^{1,2}$, Lai Wei ${ }^{1}$, Xi Xiong ${ }^{1}$, Hengyan Liu ${ }^{1}$, Jingxuan Wang ${ }^{1 *}$, Enfu

$5 \mathrm{Chen}^{3 *}$

6 Affiliations:

$7 \quad{ }^{1}$ School of Public Health and Primary Care, The Chinese University of Hong Kong, Hong Kong

8 SAR, China

$9{ }^{2}$ Shenzhen Research Institute, The Chinese University of Hong Kong, Shenzhen, China

$10{ }^{3}$ Zhejiang Province Centre for Disease Control and Prevention, Hangzhou, Zhejiang Province,

11 China

12

\section{*Address for correspondence}

14 Jingxuan Wang

RM503, School of Public Health Building, Prince of Wales Hospital, Hong Kong SAR, China

Email: jxwang@link.cuhk.edu.hk;

Enfu Chen

Zhejiang Province Centre for Disease Control and Prevention, Hangzhou, Zhejiang Province,

China

Email: enfchen@cdc.zj.cn 


\section{Abstract}

24

Background: Monitoring the reproduction number $\left(R_{t}\right)$ of the disease could help determine whether there is sustained transmission in a population, but areas with similar epidemic trends could have different transmission dynamics given the risk from imported cases varied across regions. In this study, we examined the $R_{t}$ of coronavirus disease 2019 (COVID-19) by taking different dynamics of imported cases into account and compared the transmissibility of COVID19 at different intervention periods in Hangzhou and Shenzhen.

Methods: We obtained the daily aggregated counts of laboratory-confirmed imported and local cases of COVID-19 infections in Hangzhou and Shenzhen from January 1 to March 13, 2020. Daily $R_{t}$ and piecewise $R_{t}$ before and after Wuhan lockdown were estimated, accounting for imported cases.

Results: Since the epidemic of COVID-19 in Shenzhen was dominated by imported cases, $R_{t}$ was around 0.1 to 0.7 before the Wuhan lockdown. After the lockdown of Wuhan and the initialization of measures in response to the outbreak, local transmission was well-controlled as indicated by a low estimated value of piecewise $R_{t}, 0.15$ (95\% CI: 0.09 to 0.21$)$. On the contrary, $R_{t}$ obtained for Hangzhou ranged from 1.2 to 4.9 with a piecewise $R_{t}$ of 2.55 (95\% CI: 2.13 to 2.97) before the lockdown of Wuhan due to the surge in local cases. Credits to the Wuhan lockdown and other outbreak response measures, $R_{t}$ dropped below unity in mid-February. Conclusions: Even though Shenzhen had more cases than Hangzhou, local transmission did not sustain probably due to limited transmission from imported cases owing to the reduction in local susceptibles as residents left the city during Chunyun. The lockdown measures and local outbreak responses helped reduce the local transmissibility.

Keywords: Reproduction number; COVID-19; serial interval; travel restriction 


\section{Introduction}

48 Coronaviruses are a diverse group of enveloped, positive-sense, single-stranded RNA viruses

49 that belongs to the family Coronaviridae, order Nidovirales (Richman et al., 2016). Diseases

50 caused by these viruses are zoonotic in nature, which can be transmitted between animals and

51 human (WHO, 2020a). In human, coronaviruses mainly cause respiratory tract infections with

52 severe acute respiratory syndrome (SARS) and Middle East respiratory syndrome (MERS) being

53 two notable examples. In December 2019, a novel strain of coronavirus that has not been

54 previously identified in human emerged and caused an outbreak in Wuhan, Hubei province,

55 China (WHO, 2020b). The disease responsible for the outbreak has been officially named by the

56 World Health Organization as coronavirus disease 2019 (COVID-19). Common clinical

57 manifestation of COVID-19 includes fever, fatigue, dry cough, shortness of breath and muscle

58 ache (Huang et al., 2020; Chen et al., 2020). In severe cases, COVID-19 may progress to

59 pneumonia or even death. Symptoms such as headache, dizziness, sputum production,

60 haemoptysis, diarrhoea, nausea and vomiting are less common but occasionally reported.

61 According to the Centers for Disease Control and Prevention, COVID-19 is mainly spread from

62 person-to-person via respiratory droplets, but may also be spread from contact with infected

63 surfaces or objects (CDC, n.d.). At the moment, there is no vaccine nor specific antiviral

64 treatment approved for COVID-19.

65 The current global scale health crisis triggered by COVID-19 began on 31 December 2019, the

66 day the World Health Organization was notified of several severe and unusual cases of

67 pneumonia in Wuhan. Despite the suspected source of transmission (Huanan Seafood Wholesale

68 Market) was shut down the next day, number of cases continued to expand at an alarming rate

69 (WHO, 2020c). Health experts soon identified the cause of the disease as being a novel 
coronavirus that can be spread via airborne droplets and announced the discovery on 7 January 2020. Two days later, the first related death was recorded in Wuhan; meanwhile, the disease spread outside Hubei as people travel around and outside the country, especially during "Chunyun", a 40-day period when Chinese return home for Lunar New Year reunion. On 13 January 2020, Thailand reported the first lab-confirmed case of COVID-19 outside mainland China and health authorities of 19 other countries confirmed cases over the following days (WHO, 2020d). By 31 January 2020, a total of 9,826 cases of COVID-19 were confirmed around the globe and the figure surpassed 10,000 the next day.

To halt the spread of the virus, the Chinese government imposed a complete lockdown in Wuhan and other cities in Hubei province on 23 January 2020 aiming to quarantine the epicenter of the COVID-19 outbreak (Zhao et al., 2020a). All public transportation, including buses, railways, flights, and ferry services, was suspended and residents were forbidden from leaving the city without permission from the authorities. Later, the government tightened its quarantine measures and imposed a mandatory 14-day quarantine on people returning to the capital from holidays. Meanwhile, a number of countries have imposed restrictions on entry by travellers from China in response to the epidemic. Several early investigations have shown that the shutdown of cities largely reduced the spread of infections to other cities in China and other countries around the world (Anzai et al., 2020; Tian et al., 2020; Chinazzi et al., 2020; Zhao et al., 2020b).

The basic reproduction number $\left(R_{0}\right)$ is considered to be a key indicator when studying infectious disease as it reflects the ability of infection spreading, regardless the control measures taken (You et al., 2020). Estimated $R_{0}$ of COVID-19 showed large variety, ranging from 0.48 in South Korea (Ki, 2020) to 6.30 in China (Sanche et al., 2020). The variation was affected by many factors including study region and period, modelling methods, targeted population and data 
93 source (Alimohamadi, Taghdir \& Sepandi, 2020). According to a meta-analysis of 23 studies in

94 China, the pooled $R_{0}$ of COVID-19 was 3.32 (95\% CI, 2.81-3.82) (Alimohamadi, Taghdir \&

95 Sepandi, 2020), which is much higher than that obtained in global research: Meta-analysis of 24

96 articles from 8 countries/regions estimated a reproductive number of 2.70 (95\% CI, 2.21-3.30)

97 for COVID-19 by 10 April 2020 (Liu et al., 2020). Compared to $R_{0}$, time-varying reproduction

98 number $\left(R_{t}\right)$ is a common measure for monitoring the disease evolution in a population especially

99 when some control measures have been carried out to mitigate an epidemic. It is defined as the

100 average number of secondary cases infected by a primary case at time $t$. $R_{t}$ below unity indicates

101 a single case is unable to produce more than one secondary case on average and the disease can

102 unlikely be sustained in a population over time. In this case, the outbreak can be regarded as

103 under control at time $t$ (De Serres et al., 2000). Publications on infectious diseases suggested that

104 infection prevention and control measures, timely hospitalization and quarantine, sufficient

105 healthcare resources and increased public awareness are important factors to reduce $\mathrm{R}_{\mathrm{t}}$, hence the

106 risk of infectious disease transmission (Zhao et al., 2020c). During the COVID-19 pandemic, the

107 lockdown in Wuhan was considered as an effective intervention to reduce disease transmission:

108 median daily reproduction number decreased sharply from 2.35 on 16 January to 1.05 on 30

109 January (Tian et al., 2020). Province-level, weekly-based estimation also indicated a decline in

$110 \mathrm{R}_{\mathrm{t}}$ to $<1$ in Guangdong and Zhejiang between early- and mid- February (Leung et al., 2020). A

111 more recent daily-based estimation further declared that the $R_{t}$ has reduced to $<1$ in most

112 provinces after 11 February (You et al., 2020). However, the effectiveness of travel restrictions

113 varies across cities. Studies have noted that the decline in $R_{t}$ was significantly associated with

114 passenger throughput in Hubei, ratio of imported cases, control measures, and distance from

115 Wuhan (Lau et al., 2020; Wang et al., 2020). We suggest that the effectiveness of Wuhan 
116 lockdown also varies by the dynamic of imported cases in each city. For instance, coastal cities

117 like Hangzhou and Shenzhen had large number of migrant workers returned to their hometown

118 during Spring Festival whom came back to these mega cities since early February. Separating the

119 local and imported cases is essential for the estimation of policy effectiveness. In this study, we

120 examined the transmission dynamics of COVID-19 during the first wave of epidemic and

121 compared the transmissibility of the disease at different intervention periods in Hangzhou and

122 Shenzhen, two Chinese cities with different transmission dynamics of imported cases.

\section{Methods}

\section{Setting}

125 Hangzhou and Shenzhen are two major Chinese cities in terms of economy, population and 126 transportation. Hangzhou is the capital city of Zhejiang Province and one of the most populous 127 cities in East China. In 2018, the city's resident population was 9.8 million with an estimation of 1284 million floating population (Municipality of Hangzhou, 2019). With 16,424 km length of

129 highways, well-developed railway transportation and 286 civil aviation routes between 160

130 domestic cities, the passenger traffic of Hangzhou was over 20 million in 2018 (Hangzhou

131 Statistics Bureau, n.d.). Similar to Hangzhou, Shenzhen is also a new-growing city characterized 132 by high-technology industry, large population of "migrant workers" and well-developed

133 transportation network. Located in Guangdong Province, it covers an area of around 2,000 $\mathrm{km}^{2}$

134 and is the most populous city in South China. In 2018, the number of people living in Shenzhen

135 was approximately 25.7 million, among whom less than half were long-term residents (Shenzhen

136 Bureau of Statistics, 2019). 
137 Wuhan, the source city of COVID-19, was lockdown on 23 January 2020. On the same day,

138 Hangzhou and Shenzhen declared first-level public health emergency in response to the

139 outbreak. Measures including promotion of personal hygiene, cessation of crowd gathering

140 activities, 7-day home quarantine for people from Hubei, restrictions on public transports and

141 mandatory quarantine for close contacts were adopted. On 4 February 2020, a lockdown was

142 imposed in Hangzhou and only one person per household was allowed to leave their home every

143 two days. Public gatherings such as funerals and weddings were banned; all public venues and

144 workplaces were closed. On 7 February 2020, all living communities in Shenzhen were

145 requested to lockdown in order to contain the local outbreak (Shenzhen Municipal Health

146 Commission, n.d.).

\section{Data collection}

148 We obtained the daily aggregated data of laboratory confirmed COVID-19 cases in Hangzhou

149 and Shenzhen between January 1, 2020 and March 13, 2020 from the National Health

150 Commission and other official websites (Shenzhen Municipal Health Commission, n.d.;

151 Hangzhou CDC, n.d.; NHC, n.d.) (Table S1 and S2). Date of illness onset (defined by the first

152 appearance of COVID-19 related symptoms) was used to construct the epidemic curves. For both

153 Shenzhen and Hangzhou, we followed the definition used in previous publication (Wu et al.,

154 2020) and considered two types of patients as 'imported cases': 1) those who had travelled to or

155 resided in provinces where $\geqslant 1$ confirmed COVID-19 case was reported within 14 days before

156 the onset of the disease; 2 ) those who had a travel history to provinces with $\geqslant 1$ confirmed

157 COVID-19 case, and did not have an obvious local source of infection.

158 Estimation of reproduction numbers 
159 Since the outbreak of COVID-19 in many cities outside Hubei was seeded by imported cases, the

160 estimation equation from Thompson et al. (2019), an alternative to Wallinga and Teunis (2004),

161 was used, accounting for the risk of imported cases (Chong et al., 2020).

162 The total number of reported cases $(I(t))$ on day $t(t=0,1,2,3 \ldots)$ is the sum of imported cases

$163\left(h_{I}(t)\right)$ and local cases $\left(h_{L}(t)\right)$. The total number of infections on day $t$ can be computed as

$$
\sum_{s=1}^{t} w_{s} I(t-s)=\sum_{s=1}^{t} w_{s}\left[h_{L}(t-s)+h_{I}(t-s)\right]
$$

given $w_{s}$ is a discretized probability distribution function of the serial interval of COVID-19. The expected number of local cases, $E\left[h_{L}(t)\right]$, is

$$
E\left[h_{L}(t)\right]=R_{t} \sum_{s=1}^{t} w_{s}\left[h_{L}(t-s)+h_{I}(t-s)\right] .
$$

Assume the number of local infections follows a Poisson distribution, a likelihood function, $L(\cdot)$, 169 is formed as follows

$$
L\left(R_{t}\right)=\prod_{i=t-\tau}^{t}\left(\frac{\left\{E\left[h_{L}(i)\right]\right\}^{n_{L}(i)} e^{-E\left[h_{L}(i)\right]}}{n_{L}(i) !}\right),
$$

where $n_{L}(t)$ is the observed numbers of local cases on day $t$ and $\tau$ is a smoothing parameter which is assumed to be 7 days (Thompson et al., 2019). We used Markov chain Monte Carlo (MCMC) method to estimate the $R_{t}$ series based on the observed epidemic curves. Using an early estimate of serial interval (Zhao et al., 2020b), we assumed the length of the serial interval follows a lognormal distribution with a mean of 4.4 days and a standard deviation (SD) of 3 days. A random walk Metropolis algorithm was used to obtain the posterior distributions of $R_{t}$. Step sizes were selected to obtain acceptance proportions of 20 to $40 \%$. Twenty thousand MCMC iterations were used as the burn-in period and subsequent 100,000 iterations were used to obtain the estimates. The median and 95\% credible intervals (CIs) were calculated to summarize the estimates. 
181 In addition to the time-varying reproduction number, we estimated the piecewise $R_{t}$ to compare

182 the transmissibility of COVID-19 before and after the lockdown of Wuhan (MRC Centre for

183 Global Infectious Disease Analysis, 2020). We assumed $R_{t}$ is an exponential function of a

184 baseline parameter $(\lambda)$ and an indicator variable $(X)$ for two periods (0-before lockdown and 1-

185 after lockdown):

$$
R_{t}=\lambda \exp (-\alpha X)
$$

where $\alpha$ characteristic the effect at the lockdown period. Follow similar configurations (MRC

Centre for Global Infectious Disease Analysis, 2020), we assumed $\alpha$ and $\lambda$ follow a Gamma distribution ( (hape $=0.5$, scale $=1$ ) and a normal distribution (mean=2, $\mathrm{SD}=2$ ) respectively. The posterior estimate of the piecewise $R_{t}$ and the $95 \%$ CI were determined by Bayesian non-linear modelling through Hamiltonian Monte Carlo sampling. Four chains with 2,000 of warm-up and 2,000 iterations for posterior drawing were run.

The sensitivity of results to shorter serial interval of 2.0 days (SD: 2.8) (Du et al., 2020) and longer serial interval of 7.5 days (SD: 3.4) (Li et al., 2020) was tested. The analysis in this study was carried out using software R (version 3.6.3).

\section{Results}

The temporal distribution of cases and $R_{t} \mathrm{~s}$ in Shenzhen and Hangzhou were shown in Fig 1A and 2A respectively. Shenzhen had the first imported case with illness onset on 1 January, whereas Hangzhou had the first imported case with illness onset on 13 January. From 1 January to 13 March, there were a total of 169 and 417 confirmed cases in Hangzhou and Shenzhen respectively. Both cities had their peak incidence of cases in the week between 22 and 29 January and the epidemics died out in mid-February. 
203 In general, although Shenzhen had more cases than Hangzhou, Shenzhen had a higher

204 percentage of imported cases than Hangzhou ( $83 \%$ vs 29\%). Due to the epidemic in Shenzhen

205 was dominated by imported cases, local $R_{t}$ was kept below unity through time (Fig $1 \mathrm{~B}$ and 1C).

206 In the early phase of the epidemic, $R_{t}$ was mostly maintained to be around 0.1 to 0.7 , indicating a

207 low risk of local transmission despite the rapid increase in daily number of cases before the

208 lockdown of Wuhan (i.e. on 23 January). After the lockdown of Wuhan and the initialization of

209 measures in response to the outbreak, local transmission was well-controlled as indicated by a

210 low estimated value of piecewise $R_{t}, 0.15$ (95\% CI: 0.09 to 0.21 ).

211 In contrast, $R_{t}$ was larger than unity in Hangzhou from 16 January to 7 February (Fig 2B and

212 2C). Before the lockdown of Wuhan, $R_{t}$ obtained ranged from 1.2 to 4.9 with a piecewise $R_{t} 2.55$

213 (95\% CI: 2.13 to 2.97), indicating a high risk of local transmission. Credits to the Wuhan

214 lockdown and other outbreak response measures, $R_{t}$ dropped steadily and the corresponding

215 piecewise $R_{t}$ during this period was 0.57 (95\% CI: 0.31 to 0.82 ), indicating the outbreak was 216 controlled in Hangzhou.

217 Sensitivity analysis was used to assess the robustness of estimated $R_{t}$ to different serial interval

218 durations (Figure S1). While the variation in length of serial interval did not affect the estimated

$219 R_{t} \mathrm{~s}$ in Shenzhen, a moderately increased $R_{t}$ was observed for Hangzhou during the first week

220 when a longer serial interval was assumed.

\section{Discussion}

222 Monitoring the transmission dynamics as well as the $R_{t}$ of the disease could help determine

223 whether there is sustained community transmission in a population and evaluate whether the

224 control measures taken are adequate to control the local transmission at a specific time (Nishiura 225 and Cowell, 2009; Chong et al., 2017, 2018). In this study, we estimated the imported-cases- 
226 adjusted $R_{t}$ of COVID-19 in the first wave of epidemic in Hangzhou and Shenzhen, two Chinese

227 cities with different dynamics of imported cases. According to our results, the disease

228 transmissibility in both cities gradually decreased over time especially after the lockdown of

229 Wuhan. Credits to the community lockdown, the transmission was interrupted within a short

230 period of time in Hangzhou. In line with Lai et al. (2020), inter-city travel restrictions and other

231 social distancing interventions could slow down the epidemics outside Wuhan.

232 We showed that local transmission of COVID-19 seeded by imported cases was not sustained in

233 Shenzhen and we speculated it was likely due to the decrease in number of local susceptibles

234 during Chunyun. Of the 13 million residents in Shenzhen, around 65\% of its population were

235 migrants which ranked top in China, and most of the residents have returned to their hometowns

236 starting from 10 January for celebration of Lunar New Year (Shenzhen Bureau of Statistics,

237 2019; CNN, 2020). Given the number of local susceptibles decreased as a large number of

238 residents left Shenzhen during Chunyun, fewer transmission chains could be established during

239 this period even though Shenzhen is highly connected to Wuhan for most of the times. Tian et al.

240 (2020) indicated that Chinese cities that implemented control measures before officials

241 confirmed the first case were more likely to have fewer cases. Even though the first case in

242 Shenzhen was confirmed on 19 January (illness onset on 3 January), we believe Shenzhen is an

243 exception owing to its special population characteristics. In line with Kucharski et al. (2020), the

244 case study of Shenzhen supported that introducing one to several cases to a new city may not

245 necessarily lead to an outbreak.

246 Compared to Shenzhen, Hangzhou is a highly populated city with much fewer migrants. There

247 was an estimation of 2.3 million migrant workers in Hangzhou yet 8.2 million in Shenzhen,

248 accounting for $28 \%$ and $65 \%$ of the city's total population, respectively (Zheng, Yu \& Zhang, 
249 2019; Shenzhen Bureau of Statistics, 2019). The big data on Chunyun from Baidu Map

250 (https://qianxi.baidu.com/2020/) also estimated that during January 11 and January 24, 2020, the

251 number of people leaving Shenzhen per day is on average 3 times higher than that from

252 Hangzhou. Geographically speaking, the distance from Hangzhou to Wuhan is approximately

$253336 \mathrm{~km}$ closer than that from Shenzhen. By implementing strict control measures before cases

254 emerged, local transmissions were comparatively easier to be seeded in Hangzhou. Compared to

255 the transmission dynamics in Wuhan (Kucharski et al., 2020), $R_{t}$ in Hangzhou displayed a

256 consistently declining trend staring from mid-January. This may be due to the increased

257 awareness on the use of personal protective measures against COVID-19 after noticing the

258 unknown pneumonia outbreak in Wuhan in early January through social media. Similar

259 transmission dynamics were also reported in Shaanxi province (Tang et al., 2020).

260 In this study, we showed that even though more cases were reported in Shenzhen when

261 compared to Hangzhou, they exhibited different transmission dynamics of COVID-19 when the

262 risk of imported cases was taken into account during estimation. With more local cases emerged

263 during the initial phase of the epidemic, the disease sustained in Hangzhou before the lockdown

264 of Wuhan and the initiation of outbreak response. For cities or provinces with large proportion of

265 imported cases, failing to differentiate imported cases from local cases during $R_{t}$ calculation may

266 lead to overestimation of transmissibility of COVID-19 in a local population (Xu et al., 2020),

267 hence affects the planning of mitigation measures. The importance of accounting for imported

268 cases has been demonstrated in another study that looked into MERS in Saudi Arabia

269 (Thompson et al., 2019).

270 Previous scholars discussed the importance of Chunyun in COVID-19 transmission. On one

271 hand, as migrants travelled long distance and returned to their birthplace from high-risk 
272 megacities, their travel brings pressure to disease control in their birthplaces which are usually

273 economically and socially undeveloped regions with high proportions of elderly and less

274 experience in epidemic management. On the other hand, outflow of migrant workers leads to

275 decreased urban population density and lowers the risk of disease spread in migrant cities (Chen

276 et al., 2020; Leung et al., 2020). Although we only have data from Shenzhen and Hangzhou, a

277 network analysis of 22 Chinese migrant cities noted the time-varying risk of COVID-19

278 transmission in Shenzhen is very similar to that in many other migrant cities such as Guangzhou,

279 Beijing, Shanghai and Chongqing (Fan et al., 2020).

280 Our study has several major limitations. Firstly, the definition of the import cases was relied on

281 the epidemiological investigation of travel history and there was a possibility that a case infected

282 locally but having a travel history to other provinces 14 days prior to symptoms onset. The

283 misclassification of the imported cases would underestimate the local transmissibility in our

284 study. In addition, recent studies have successively reported that some patients infected with

285 COVID-19 might infect others before their symptom onset (Du et al., 2020; Nishiura et al.,

286 2020). Disease transmission during the pre-symptomatic stage implies the possibility of having a

287 negative value of serial interval. This would affect the formulation of estimation equation since

288 the distributional assumption did not gave it a corresponding probability. Alternatively,

289 generation interval could be used but it is usually hard to be estimated since the onset of

290 infectiousness is impossible to observe. Nevertheless, as demonstrated in the sensitivity analysis,

291 variation in serial interval within a reasonable range would not affect our main conclusion.

292 Another limitation is the underreporting of confirmed cases due to unavailability of virological

293 testing during the early stage of epidemic. According to an early investigation (Wu et al., 2020),

294 an estimate of 75 thousand individuals were found to be infected in Wuhan as of 25 January and 
295 we believe similar underreporting was likely to occur in our setting. With data on reporting rates

296 and serological surveillance available in detail, our analytic frame can be extended to a more

297 complex context that incorporates these factors. In addition, our estimates shall be refined if

298 more updated knowledge of the pathogen is available.

\section{Conclusions}

300 In conclusion, we showed the lockdown measures and local outbreak responses helped reduce

301 the potential of local transmission in Hangzhou and Shenzhen. The low transmission intensity of

302 COVID-19 in Shenzhen in early January was likely due to the decrease in number of local

303 susceptibles as residents left the city during Chunyun. We also highlighted that, given the

304 variation in imported cases, cities with similar epidemic trend could have different transmission

305 dynamics. 


\section{Acknowledgments}

309 We thank the physicians and staffs at Hangzhou, Huzhou, Jiaxing Wenzhou, Shaoxing, Ningbo,

310 Quzhou, Jinhua, Zhoushan, Lishui, Taizhou Municipal Center for Disease Control and

311 Prevention for their support and assistance with this investigation.

312

313

314

315

316

317

318

319

320

321

322

323

324

325

326

327 
329

330

Alimohamadi Y, Taghdir M, Sepandi M. The estimate of the basic reproduction number for novel coronavirus disease (COVID-19): a systematic review and meta-analysis. Journal of Preventive Medicine and Public Health. 2020.

Anzai A, Kobayashi T, Linton NM, Kinoshita R, Hayashi K, Suzuki A, Yang Y, Jung SM, Miyama T, Akhmetzhanov AR, Nishiura H. Assessing the impact of reduced travel on exportation dynamics of novel coronavirus infection (COVID-19). Journal of clinical medicine. $2020 \mathrm{Feb} ; 9(2): 601$.

Centers for Disease Control and Prevention (CDC). About Coronavirus Disease 2019 (COVID19). Available from: https://www.cdc.gov/coronavirus/2019-ncov/about/index.html [accessed 22 Feb 2020]

Chen S, Yang J, Yang W, Wang C, Bärnighausen T. COVID-19 control in China during mass population movements at New Year. The Lancet. 2020 Mar 7;395(10226):764-6.

Chen N, Zhou M, Dong X, Qu J, Gong F, Han Y, Qiu Y, Wang J, Liu Y, Wei Y, Yu T. Epidemiological and clinical characteristics of 99 cases of 2019 novel coronavirus pneumonia in Wuhan, China: a descriptive study. The Lancet. 2020 Feb 15;395(10223):507-13.

Chinazzi M, Davis JT, Ajelli M, Gioannini C, Litvinova M, Merler S, y Piontti AP, Mu K, Rossi L, Sun K, Viboud C. The effect of travel restrictions on the spread of the 2019 novel coronavirus (COVID-19) outbreak. Science. 2020;368(6489):395-400..

Chong KC, Cheng W, Zhao S, Ling F, Mohammad KN, Wang MH, Zee BC, Wei L, Xiong X, Liu H, Wang J. Monitoring disease transmissibility of 2019 novel coronavirus disease in Zhejiang, China. International Journal of Infectious Diseases. 2020;96:128-30.

Chong KC, Hu P, Lau S, Jia KM, Liang W, Wang MH, Zee BC, Sun R, Zheng H. Monitoring the age-specificity of measles transmissions during 2009-2016 in Southern China. PloS one. 2018 Oct 8;13(10):e0205339.

Chong KC, Zhang C, Zee BC, Luo T, Wang L, Tam GC, Jia KM, Sun R, Wang MH, Guan X. Interpreting the transmissibility of measles in two different post periods of supplementary immunization activities in Hubei, China. Vaccine. 2017 Feb 15;35(7):1024-9.

CNN. 3 billion journeys: World's biggest human migration begins in China. 2020. Available from: https://edition.cnn.com/travel/article/chunyun-2020-lunar-new-year-travel-rushchina/index.html [accessed 15 March 2020]

De Serres G, Gay NJ, Farrington CP. Epidemiology of transmissible diseases after elimination. Am J Epidemiol 2000;151(11):1039-48.

Du Z, Xu X, Wu Y, Wang L, Cowling BJ, Meyers LA. Serial interval of COVID-19 among publicly reported confirmed cases. Emerging infectious diseases. 2020 ;26(6):1341. Fan C, Cai T, Gai Z, Wu Y. The relationship between the migrant population's migration network and the risk of COVID-19 transmission in China-Empirical analysis and prediction in prefecture-level cities. International Journal of Environmental Research and Public Health. 2020 Jan;17(8):2630. 
367 Hangzhou Center for Disease Control and Prevention (Hangzhou CDC). Statistics. Available

368 from: http://www.hzcdc.net/jbkz/jbkz01.htm [accessed 15 March 2020]

369 Hangzhou Statistics Bureau. Transportation, post and telecommunication. In: Hangzhou

370 Statistical Yearbook 2018. China Statistics Press. [accessed 9 March 2020]

371 Hien Lau, Veria Khosrawipour, Piotr Kocbach, Agata Mikolajczyk, Justyna Schubert, Jacek

372 Bania, Tanja Khosrawipour, The positive impact of lockdown in Wuhan on containing the

373 COVID-19 outbreak in China, Journal of Travel Medicine, Volume 27, Issue 3, April 2020,

374 taaa037, https://doi.org/10.1093/jtm/taaa037

375 Huang C, Wang Y, Li X, Ren L, Zhao J, Hu Y, Zhang L, Fan G, Xu J, Gu X, Cheng Z. Clinical

376 features of patients infected with 2019 novel coronavirus in Wuhan, China. The lancet. 2020 Feb

377 15;395(10223):497-506.

378 Jian Wu, Jun Liu, Xinguo Zhao, Chengyuan Liu, Wei Wang, Dawei Wang, Wei Xu, Chunyu

379 Zhang, Jiong Yu, Bin Jiang, Hongcui Cao, Lanjuan Li, Clinical Characteristics of Imported

380 Cases of Coronavirus Disease 2019 (COVID-19) in Jiangsu Province: A Multicenter Descriptive

381 Study, Clinical Infectious Diseases, Volume 71, Issue 15, 1 August 2020, Pages 706-

382 712, https://doi.org/10.1093/cid/ciaa199

$383 \mathrm{Ki} \mathrm{M}$. Epidemiologic characteristics of early cases with 2019 novel coronavirus (2019-nCoV)

384 disease in Korea. Epidemiology and health. 2020 Feb 9;42.

385 Kucharski AJ, Russell TW, Diamond C, Liu Y, Edmunds J, Funk S, Eggo RM, Sun F, Jit M,

386 Munday JD, Davies N. Early dynamics of transmission and control of COVID-19: a

387 mathematical modelling study. The lancet infectious diseases. 2020 Mar 11.

388 Lai S, Ruktanonchai NW, Zhou L, Prosper O, Luo W, Floyd JR, Wesolowski A, Santillana M, 389 Zhang C, Du X, Yu H, Tatem AJ. Effect of non-pharmaceutical interventions to contain COVID39019 in China. Nature. 2020;10.1038/s41586-020-2293-X.

391 Leung K, Wu JT, Liu D, Leung GM. First-wave COVID-19 transmissibility and severity in 392 China outside Hubei after control measures, and second-wave scenario planning: a modelling 393 impact assessment. The Lancet. 2020 Apr 8.

394 Li Q, Guan X, Wu P, Wang X, Zhou L, Tong Y, Ren R, Leung KS, Lau EH, Wong JY, Xing X. 395 Early transmission dynamics in Wuhan, China, of novel coronavirus-infected pneumonia. New 396 England Journal of Medicine. 2020 Jan 29.

397 Liu Y, Gayle AA, Wilder-Smith A, Rocklöv J. The reproductive number of COVID-19 is higher 398 compared to SARS coronavirus. Journal of travel medicine. 2020 Mar 13.

399 MRC Centre for Global Infectious Disease Analysis. Report 13 - Estimating the number of 400 infections and the impact of non-pharmaceutical interventions on COVID-19 in 11 European 401 countries. 2020. Available from: https://www.imperial.ac.uk/mrc-global-infectious-disease402 analysis/covid-19/report-13-europe-npi-impact/ [accessed 15 March 2020]

403 Municipality of Hangzhou. Bulletin on 2018 Hangzhou population. In: "China Hangzhou" 404 Government Portal. 2019. Available from:

405 http://www.hangzhou.gov.cn/art/2019/2/14/art_805865_30213801.html [accessed 9 March 2020] 
406 National Health Commission of the People's Republic of China (NHC). COVID-19 prevention

407 and Control. Available from: http://www.nhc.gov.cn/xcs/yqtb/list gzbd.shtml [accessed 15

408 March 2020]

409 Nishiura H, Linton NM, Akhmetzhanov AR. Serial interval of novel coronavirus (COVID-19)

410 infections. Int J Infect Dis 2020;93:284-286.

411 Nishiura H., Cowell G. The Effective Reproduction Number as a Prelude to Statistical

412 Estimation of Time-Dependent Epidemic Trends. In: Chowell G, Hyman JM, Bettencourt LMA,

413 Castillo-Chavez C, editors. Mathematical and Statistical Estimation Approaches in

414 Epidemiology. Dordrecht: Springer; 2009. p. 103-121.

415 Richman DD, Whitley RJ, Hayden FG. Clinical virology, 4th edn. Washington: ASM Press;

4162016.

417 Sanche S, Lin YT, Xu C, Romero-Severson E, Hengartner NW, Ke R. The novel coronavirus, 418 2019-nCoV, is highly contagious and more infectious than initially estimated. arXiv preprint 419 arXiv:2002.03268. 2020 Feb 9.

420 Shenzhen Bureau of Statistics. Shenzhen statistical yearbook. 2019. Available from:

421 http://tjj.sz.gov.cn/zwgk/zfxxgkml/tjsj/tjnj/201912/t20191230 18956670.htm [accessed 9 March

422 2020]

423 Shenzhen Municipal Health Commission [Internet]. COVID-19 News. Available from:

424 http://wjw.sz.gov.cn/ [accessed 9 March 2020]

425 Tang S, Tang B, Bragazzi NL, Xia F, Li T, He S, Ren P, Wang X, Peng Z, Xiao Y, Wu J.

426 Stochastic discrete epidemic modeling of COVID-19 transmission in the Province of Shaanxi

427 incorporating public health intervention and case importation. medRxiv. 2020 Jan 1.

428 Thompson RN, Stockwin JE, van Gaalen RD, Polonsky JA, Kamvar ZN, Demarsh PA, 429 Dahlqwist E, Li S, Miguel E, Jombart T, Lessler J. Improved inference of time-varying 430 reproduction numbers during infectious disease outbreaks. Epidemics. 2019 Dec 1;29:100356.

431 Tian H, Liu Y, Li Y, Kraemer MU, Chen B, Wu CH, Cai J, Li B, Xu B, Yang Q, Wang B. Early 432 evaluation of transmission control measures in response to the 2019 novel coronavirus outbreak 433 in China. medRxiv. 2020 Jan 1.

434 Wallinga J, Teunis P. Different epidemic curves for severe acute respiratory syndrome reveal 435 similar impacts of control measures. Am J Epidemiol 2004;160(6):509-16.

436 Wang K, Zhao S, Li H, Song Y, Wang L, Wang MH, Peng Z, Li H, He D. Real-time estimation 437 of the reproduction number of the novel coronavirus disease (COVID-19) in China in 2020 based 438 on incidence data. Annals of Translational Medicine. 2020 Jun;8(11).

439 World Health Organization (WHO). Coronavirus. 2020a. Available from:

440 https://www.who.int/health-topics/coronavirus [accessed 22 Feb 2020]

441 World Health Organization (WHO). Novel coronavirus - China. Jan 12, 2020. 2020b. Available 442 from: https://www.who.int/csr/don/12-january-2020-novel-coronavirus-china/en/ [accessed 22

443 Feb 2020] 
444 World Health Organization (WHO). Novel Coronavirus (2019-nCoV) Situation Report - 1. 445 2020c. Available from: https://www.who.int/docs/default-source/coronaviruse/situation446 reports/20200121-sitrep-1-2019-ncov.pdf?sfvrsn=20a99c10_4 [accessed 22 Feb 2020]

447 World Health Organization (WHO). Novel Coronavirus (2019-nCoV) Situation Report - 11. 448 2020d. Available from: https://www.who.int/docs/default-source/coronaviruse/situation449 reports/20200131-sitrep-11-ncov.pdf?sfvrsn=de7c0f7_4 [accessed 22 Feb 2020]

$450 \mathrm{Wu}$ JT, Leung $\mathrm{K}$, Leung GM. Nowcasting and forecasting the potential domestic and 451 international spread of the $2019-\mathrm{nCoV}$ outbreak originating in Wuhan, China: a modelling study. 452 Lancet 2020;395(10225):689-97.

453 Xu L, Yuan J, Zhang Y, Zhang G, Lu F, Su J, Qu J. Highland of COVID-19 outside Hubei: 454 epidemic characteristics, control and projections of Wenzhou, China. medRxiv. 2020 Jan 1.

455 You C, Deng Y, Hu W, Sun J, Lin Q, Zhou F, Pang CH, Zhang Y, Chen Z, Zhou XH. Estimation 456 of the time-varying reproduction number of COVID-19 outbreak in China. International Journal 457 of Hygiene and Environmental Health. 2020 May 11:113555.

458 Zhao S, Stone L, Gao D, Musa SS, Chong MK, He D, Wang MH. Imitation dynamics in the 459 mitigation of the novel coronavirus disease (COVID-19) outbreak in Wuhan, China from 2019 to 460 2020. Annals of Translational Medicine. 2020a Apr;8(7).

461 Zhao S, Gao D, Zhuang Z, Chong MK, Cai Y, Ran J, Cao P, Wang K, Lou Y, Wang W, Yang L. 462 Estimating the serial interval of the novel coronavirus disease (COVID-19): A statistical analysis 463 using the public data in Hong Kong from January 16 to February 15, 2020. medRxiv. 2020b.

464 [doi: https://doi.org/10.1101/2020.02.21.20026559]

465 Zhao S, Tang X, Liang X, Chong MK, Ran J, Musa SS, Yang G, Cao P, Wang K, Zee BC, Wang 466 X. Modelling the Measles Outbreak at Hong Kong International Airport in 2019: A Data-Driven 467 Analysis on the Effects of Timely Reporting and Public Awareness. Infection and Drug 468 Resistance. 2020c;13:1851.

469 Zeng $\mathrm{H}, \mathrm{Yu} \mathrm{X}$, Zhang J. Urban village demolition, migrant workers' rental costs and housing 470 choices: Evidence from Hangzhou, China. Cities. 2019 Nov 1;94:70-9. 


\section{Figure Titles and Legends}

473 Figure 1. Temporal distribution of cases and $\boldsymbol{R}_{t \boldsymbol{s}}$ in Shenzhen (A) Epidemic trend, (B)

474 Estimate (solid line) and 95\% credible intervals (dotted lines) of the time-varying reproduction

475 number $\left(R_{t}\right)$, and (C) Estimate and 95\% credible band of the piecewise $R_{t}$ in Shenzhen

476 Figure 2. Temporal distribution of cases and $\boldsymbol{R}_{t s}$ in Hangzhou (A) Epidemic trend, (B)

477 Estimate (solid line) and 95\% credible intervals (dotted lines) of the time-varying reproduction

478 number $\left(R_{t}\right)$, and (C) Estimate and 95\% credible band of the piecewise $R_{t}$ in Hangzhou

479

480 Supplementary materials

481 Figure S1. Time-varying reproduction number $\left(R_{t}\right)$ (left panel) and piecewise $R_{t}$ (right panel)

482 assuming a shorter (A-D) and longer serial interval (E-H) in Shenzhen (A, B, E, and F) and

483 Hangzhou (C, D, G, and H) respectively.

484 Table S1. Number of local and imported cases by illness onset date in Hangzhou, China

485 Table S2. Number of local and imported cases by illness onset date in Shenzhen, China

486 
Figure 1

Temporal distribution of cases and $R_{t s}$ in Shenzhen

(A) Epidemic trend, (B) Estimate (solid line) and 95\% credible intervals (dotted lines) of the time-varying reproduction number $\left(R_{t}\right)$, and $(C)$ Estimate and $95 \%$ credible band of the piecewise $R_{t}$ in Shenzhen 


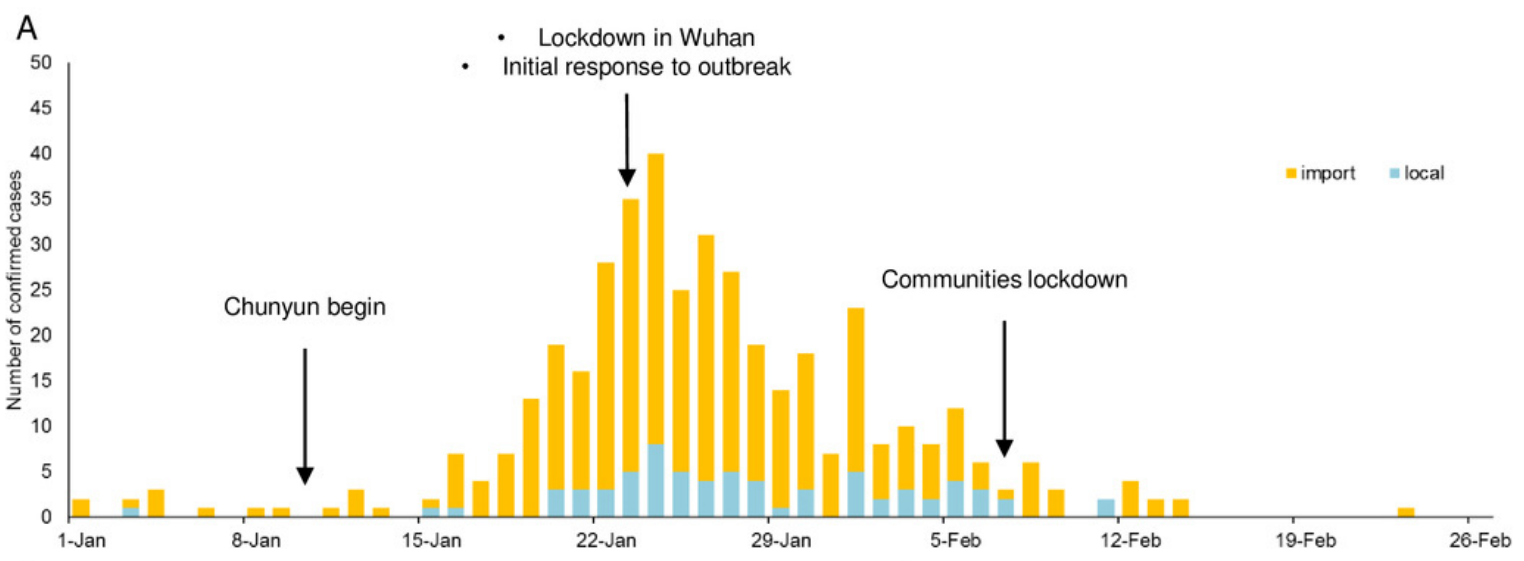

B

Symptom onset date

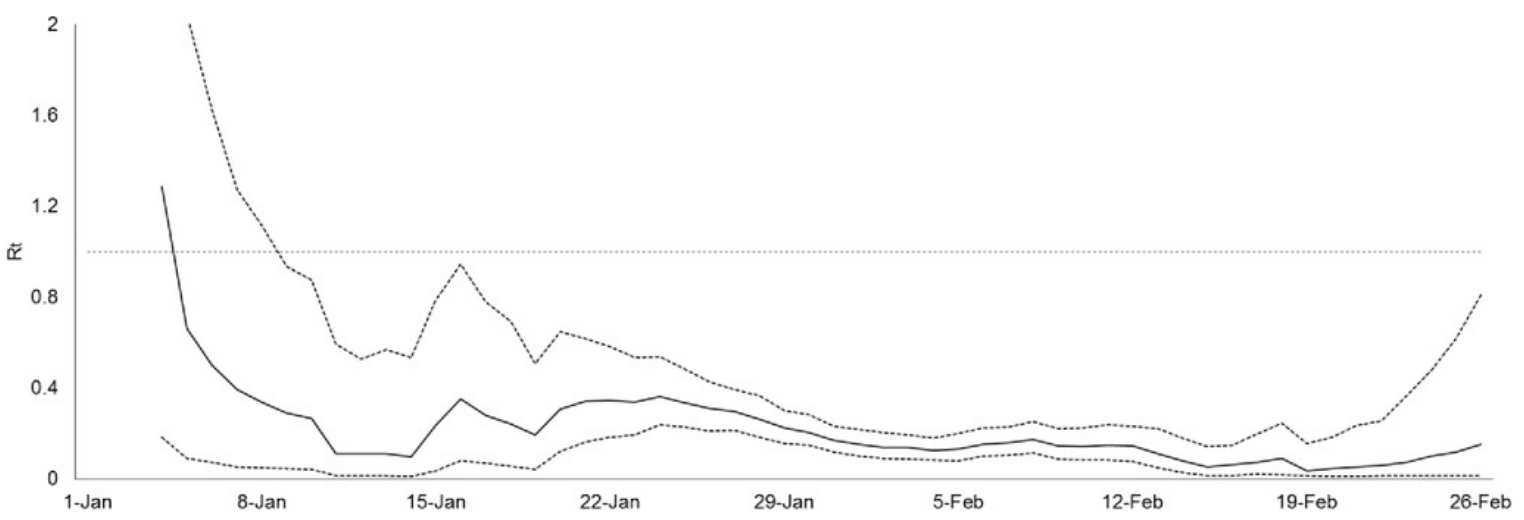

C

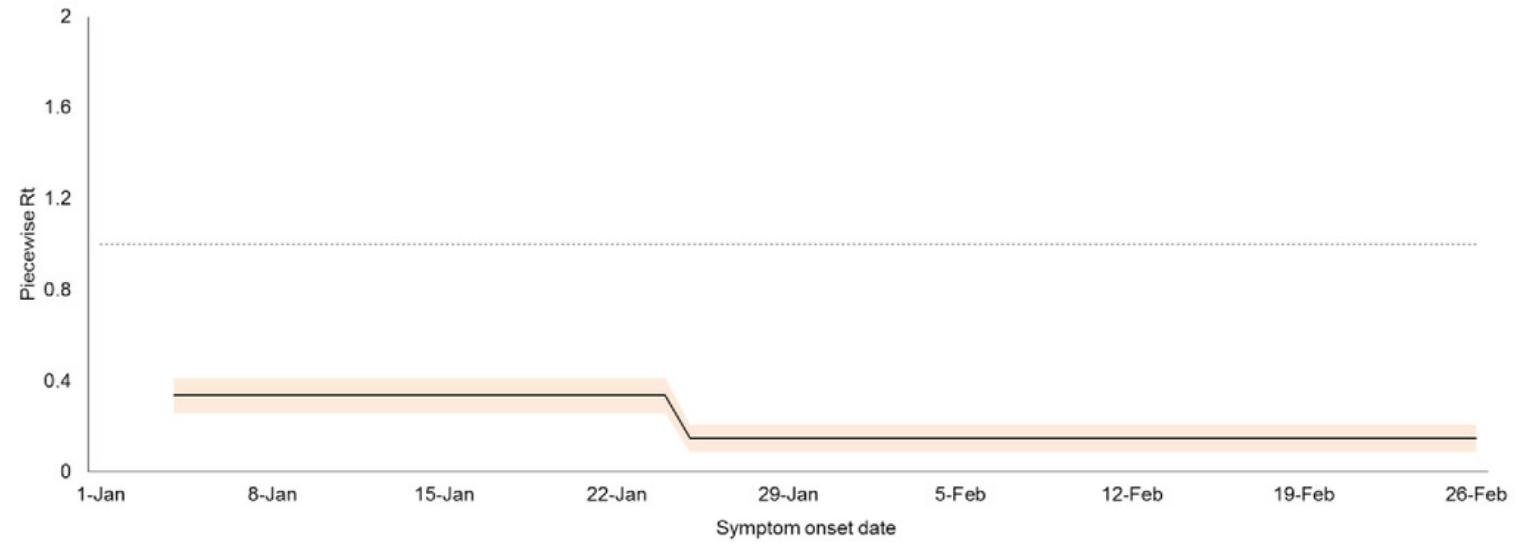


Figure 2

Temporal distribution of cases and $R_{t s}$ in Hangzhou

(A) Epidemic trend, (B) Estimate (solid line) and 95\% credible intervals (dotted lines) of the time-varying reproduction number $\left(R_{t}\right)$, and $(C)$ Estimate and $95 \%$ credible band of the piecewise $R_{t}$ in Hangzhou 

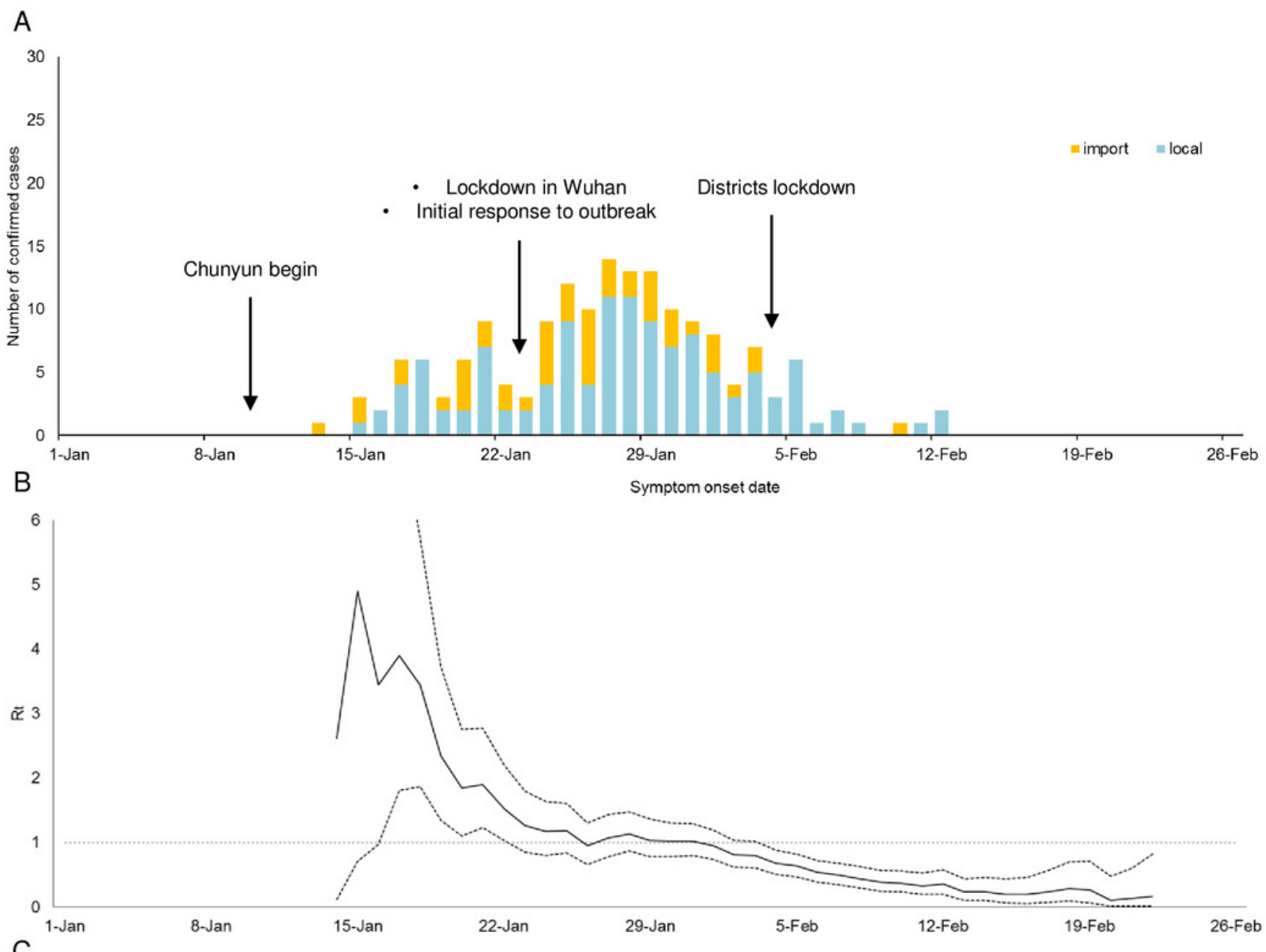

C

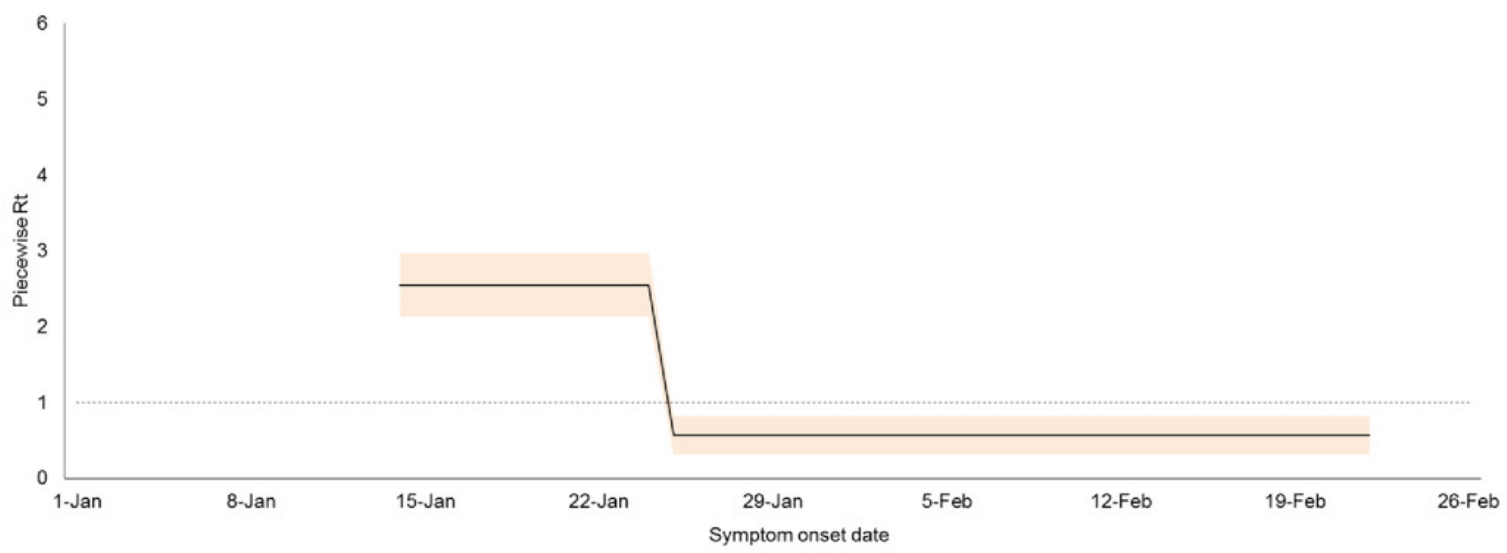

